\section{PWE-023 COULD FAECAL IMMUNOCHEMICAL TESTS FOR HAEMOGLOBIN (FIT) CHANGE SURVEILLANCE OF PEOPLE WITH INTERMEDIATE RISK ADENOMAS?}

doi:10.1136/gutjnl-2013-304907.312

1." Kralj-Hans, ' J Martin, ${ }^{2} \mathrm{~S}$ Pearson, ${ }^{2} \mathrm{C}$ Piggott, ${ }^{3} \mathrm{H}$ Bowyer, ${ }^{3} \mathrm{G}$ Vart, ${ }^{3} \mathrm{C}$ von Wagner, ${ }^{3} \mathrm{~J}$ Wardle, ${ }^{4} \mathrm{~S}$ Halloran, 'W Atkin. 'Department of Surgery and Cancer, Imperial College London, London; ${ }^{2}$ NHS Bowel Cancer Screening Programme, Southern Hub, Guildford; ${ }^{3}$ Department of Epidemiology and Public Health, University College London, London; ${ }^{4}$ University of Surrey, Guildford, UK

Introduction The NHS Bowel Cancer Screening Programme (BCSP) in England uses a biennial guaiac faecal occult blood test and identifies intermediate risk (IR) adenomas in approximately 5,000 people a year who are then offered colonoscopic surveillance. A noncolonoscopic approach to surveillance in this group may be an attractive alternative which could reduce demand on this expensive and limited resource.

Methods In the 'FIT for Follow-Up' study we aim to determine the three-year programme sensitivity of annual FIT for detection of advanced adenomas (an adenoma $>10 \mathrm{~mm}$, or one with tubulovillous or villous histology, or with high grade dysplasia) or colorectal cancer (CRC), using surveillance colonoscopy as the reference standard. Eligible participants are people aged 60-71 years, who have recently been diagnosed with IR adenomas in the BCSP, and are awaiting their first surveillance colonoscopy at three years. They are invited to complete a FIT test (Eiken Chemicals, Inc.) annually in the three year interval between screening and surveillance colonoscopy. Those who test positive (> $200 \mathrm{ng}$ haemoglobin/mL buffer; OC Sensor) are offered immediate colonoscopy; those who test negative are offered another FIT test a year later. In total, eligible participants could complete three FIT tests prior to their first surveillance colonoscopy. Our sample size is 5,200 participants completing a FIT test within a year of their screening colonoscopy. The study will also assess the acceptability and cost-effectiveness of annual FIT as an alternative to three-yearly surveillance colonoscopy.

Results In the first 10 months of the study, 2,512 people $(65.5 \%$ men) consented to take part and completed their first FIT test. The positivity rate in this group is $5.4 \%$ (6.1\% in men and $3.3 \%$ in women) and only $7 \%$ of people testing positive declined to have their three-year surveillance examination brought forward. In FIT positive subjects who underwent colonoscopy, $24.7 \%$ had advanced adenomas and one participant had a cancer in the proximal colon.

Virtually all study participants were satisfied with their experience of completing their first FIT kit (95.8\%), and would be happy to do so again (94.7\%). The majority of participants (over $80 \%$ ) believed that participating in the study had reduced their chances of having bowel cancer.

Conclusion At this early stage in the study we can report that FIT is well accepted by participants and the yield at colonoscopy in those testing positive is high.

Disclosure of Interest None Declared.

\section{PWE-024 PREVALENCE OF CHRONIC GASTROINTESTINAL SIDE EFFECTS FOLLOWING PELVIC RADIOTHERAPY IN A REGIONAL ONCOLOGY CENTRE}

doi:10.1136/gutjnl-2013-304907.313

${ }^{1} \mathrm{~L}$ Abraham, ${ }^{2} \mathrm{~J}$ Green, ${ }^{2, *} \mathrm{~J}$ Turner. ${ }^{1}$ Cardiff University School of Medicine; ${ }^{2}$ Gastroenterology, University Hospital Llandough, Cardiff, UK

Introduction The chronic gastrointestinal (GI) side effects of pelvic radiotherapy (RT) can significantly impact on patients quality of life. The survival rates of many cancers are improving due to advances in multimodality therapies; consequently there is an emergent population of patients living with the long term side effects of their treatment. Our aim was to determine the prevalence of GI symptoms amongst patients treated with pelvic RT in a regional oncology centre (Velindre Hospital, Cardiff).

Methods A search of the Cancer Network Information System Cymru (CANISC) database identified patients who had received radical radiotherapy for gynaecological, colorectal or urological malignancy between $1 / 1 / 08$ and $30 / 6 / 08$. This period was chosen to allow a reasonable time to lapse for the majority of chronic radiotherapy side effects to be present. Case notes were reviewed to ascertain patient demographics, treatment plans and symptoms reported. GI symptoms reported $\geq 3$ months after the end of RT were included. Rates of toxicity were compared to those reported in BSG guidance. ${ }^{1}$

Results 295 patients (218 male) received radical pelvic RT. GI symptoms were recorded in $31 \%$ of patients. $9 \%$ described more than one symptom up to 4 years post-treatment. No particular malignancy site, age or gender experienced significantly more GI symptoms ( $p>0.05$, Fisher exact, two tailed). Rates of potential toxicity were comparable to the BSG guidance for gynaecological ( $37.3 \%$ vs $40 \%$ respectively) and urological (28.7\% vs $30 \%$ ) malignancy. ${ }^{1}$ GI symptoms in the colorectal group were less than the BSG guidance ( 31.3 vs $66 \%>$ short course RT and $50 \%>$ chemo-radiation and surgery). The most prevalent symptoms were diarrhoea amongst gynaecological and colorectal patients and rectal bleeding amongst patients who had received treatment for prostatic carcinoma.

Conclusion A significant proportion of patients experience GI symptoms, which may be due to pelvic RT. We are likely to have underestimated reported symptoms, as data was taken from case notes rather than prospective patient questionnaires. Our overall prevalence and distribution of chronic GI symptoms in radical pelvic radiotherapy patients is similar to that stated in the literature. The majority of existing data on this subject is from one centre (Royal Marsden Hospital). As our data is similar to their reported data it is likely that it is a true reflection of the problem nationally. Only a minority of patients have previously been referred to gastroenterologists - this has increased and will continue to do so because of awareness raised by the BSG guidance.

Disclosure of Interest None Declared.

\section{REFERENCE}

1. Andreyev HJN, et al. Practice guidance on the management of acute and chronic gastrointestinal problems arising as a result of treatment for cancer. Gut 2012 61(2):179-92.

\section{PWE-025 ASSESSING THE PERCENT OF DAYS LINACLOTIDE IMPROVED ABDOMINAL SYMPTOMS AND STOOL FREOUENCY IN PATIENTS WITH IRRITABLE BOWEL SYNDROME WITH CONSTIPATION (IBS-C): POOLED ANALYSIS OF 2 PHASE 3 TRIALS}

doi:10.1136/gutjnl-2013-304907.314

'S S Rao, ${ }^{2} \mathrm{~L}$ Chang, ${ }^{3} \mathrm{X}$ Hao, ${ }^{3} \mathrm{~B}$ Lavins, ${ }^{4} \mathrm{~S} J$ Shiff, ${ }^{4} \mathrm{X}$ Cao, ${ }^{4} \mathrm{H}$ Schneier, ${ }^{3} \mathrm{M}$ Currie, 3."J Johnston. 'Georgia Health Sciences University, Augusta; ${ }^{2}$ David Geffen School of Medicine at UCLA, Los Angeles; ${ }^{3}$ Ironwood Pharmaceuticals, Cambridge; ${ }^{4}$ Forest Research Institute, Jersey City, United States

Introduction Linaclotide (LIN), a minimally absorbed guanylate cyclase $\mathrm{C}$ agonist (GCCA), improved abdominal symptoms and bowel movement (BM) frequency in patients (pts) with IBS-C in 2 Phase 3 trials. This post-hoc analysis determined the \% of days pts reported improvements in abdominal symptoms and BMs with LIN or placebo $(\mathrm{PBO})$ treatment.

Methods In 2 Phase 3 trials, pts with IBS-C (Rome II criteria) were randomised to LIN 290 - $\mu$ g qd po or PBO. Using pooled intentto-treat (ITT) data from the $1^{\text {st }} 12 \mathrm{wks}$ of both trials, we determined 
(for pts with an average baseline score $\geq 3$ out of 10 for each respective parameter) 1) $\%$ of days with $\geq 30 \%$ improvement in either abdominal pain, discomfort, bloating, cramping or fullness and 2) $\%$ of days with a spontaneous BM (SBM) or a complete SBM (CSBM).

Results The pooled ITT population included 797 PBO- and 805 LIN-treated pts. During the 2-wk Baseline Period, pts had an SBM on a mean of $24 \%$ of days and a CSBM on a mean of $3 \%$ of days. Pts in subgroups with an average baseline abdominal symptom score $\geq 3$ experienced $\geq 30 \%$ improvement in the specific abdominal symptom on a significantly greater \% of days for LIN vs PBO (Table). LINtreated pts had $\geq 30 \%$ improvement in abdominal pain for $55 \%$ of days vs $41 \%$ for PBO ( $p<0.0001)$. Results were similar for other abdominal symptoms, with LIN-treated pts achieving $\geq 30 \%$ improvement for $\sim 50 \%$ of days during the Treatment Period; PBO pts met the threshold on $33-42 \%$ of days. LIN also significantly increased the \% of days with SBM/CSBM vs PBO (Table).

\section{Abstract PWE-025 Table}

\begin{tabular}{lccccc}
\hline & $\mathbf{n}$ & PBO & LIN & $\Delta$ & P-value $^{\text {a }}$ \\
\hline \% of days with $\geq 30 \%$ improvement in abdominal symptoms & & & \\
Pain $^{\mathrm{b}}$ & 1602 & 41 & 55 & 13 & $<0.0001$ \\
Discomfort $^{\mathrm{c}}$ & 1589 & 38 & 52 & 14 & $<0.0001$ \\
Bloating $^{\mathrm{c}}$ & 1574 & 33 & 48 & 15 & $<0.0001$ \\
Cramping $^{\mathrm{c}}$ & 1457 & 42 & 55 & 13 & $<0.0001$ \\
Fullness $^{\mathrm{c}}$ & 1583 & 33 & 49 & 15 & $<0.0001$ \\
\% of days with SBM/CSBM & & & & & \\
SBM $^{\text {CSBM }}$ & 1602 & 37 & 54 & 17 & $<0.0001$ \\
\hline
\end{tabular}

SBM, spontaneous bowel movement; CSBM, complete SBM aWilcoxon Rank Sum test; ${ }^{b}$ mean abdominal pain score $\geq 3$ during the Baseline Period; ' $a v e r a g e$ baseline score $\geq 3$ for that symptom

Conclusion LIN improved abdominal symptoms (pain, discomfort, bloating, fullness and cramping) by $\geq 30 \%$ on $\sim 50 \%$ of treatment days and significantly increased the \% of days with SBM/ CSBM. Thus, LIN relieved key IBS-C symptoms and increased the $\%$ of days with symptom improvement. These data provide estimates for results of treatment with LIN that clinicians and pts may anticipate. Supported by Ironwood Pharmaceuticals Inc and by Forest Laboratories, Inc. Editing assistance was provided by Complete Medical Communications, funded by Almirall

Disclosure of Interest S. Rao Grant/Research Support from: SmartPill Corporation, Forest Laboratories and Ironwood Pharmaceuticals, Conflict with: Advisory board for Forest Laboratories and Ironwood Pharmaceuticals. Sits on Advisory Board Smart Pill Corporation, AMS, AstraZeneca and Asubio Pharmaceuticals, L. Chang Grant/Research Support from: Ironwood Pharmaceuticals and Forest Laboratories, Consultant for: Ironwood Pharmaceuticals and Forest Laboratories, X. Hao Shareholder of: Ironwood Pharmaceuticals, Employee of: Ironwood Pharmaceuticals, B. Lavins Shareholder of: Ironwood Pharmaceuticals, Employee of: Ironwood Pharmaceuticals, S. Shiff Shareholder of: Forest Laboratories, Employee of: Forest Laboratories, X. Cao Employee of: Forest Laboratories, H. Schneier Shareholder of: Forest Laboratories, Employee of: Forest Laboratories, M. Currie Shareholder of: Ironwood Pharmaceuticals, Employee of: Ironwood Pharmaceuticals, J. Johnston Shareholder of: Ironwood Pharmaceuticals, Employee of: Ironwood Pharmaceuticals

\section{PWE-026 EFFECTS OF 26 WEEKS OF LINACLOTIDE TREATMENT ON ADEQUATE RELIEF AND IBS SEVERITY IN PATIENTS WITH IRRITABLE BOWEL SYNDROME WITH CONSTIPATION (IBS-C)}

doi:10.1136/gutjnl-2013-304907.315
${ }^{1} \mathrm{E}$ M Quigley, ${ }^{2} \mathrm{~W} D$ Chey, ${ }^{3 B}$ Lavins, ${ }^{3} \mathrm{M}$ Baird, ${ }^{3} \mathrm{D}$ Fitch, ${ }^{3} \mathrm{X}$ Hao, ${ }^{4} \mathrm{~K}$ Shi, ${ }^{3} \mathrm{M}$ Currie, ${ }^{4} \mathrm{H}$ Schneier, ${ }^{3, *}$ J Johnston. 'University College Cork, Cork, Ireland; '2University of Michigan Health System, Ann Arbor; ${ }^{3}$ Ironwood Pharmaceuticals, Cambridge; ${ }^{4}$ Forest Research Institute, Jersey City, United States

Introduction There have been few long-term studies evaluating treatments for IBS. Linaclotide (LIN), a 14 amino acid, minimally absorbed, guanylate cyclase $\mathrm{C}$ agonist (GCCA), was evaluated in 2 Phase 3 trials in patients (pts) with IBS-C, including a double-blind trial with an extended $26 \mathrm{wk}$ Treatment Period, in which statistically significant improvements in abdominal pain and complete spontaneous bowel movement (CSBM) frequency were sustained for up to $26 \mathrm{wks}$. In a subsequent analysis of this study, we assessed the effects of 26 wks of LIN treatment on adequate relief and IBS severity and the relationship between long-term improvement in adequate relief with improvements in abdominal pain and CSBM frequency.

Methods Adult pts with IBS-C (Rome II criteria) were randomised to LIN $290 \mu \mathrm{g}$ or placebo (PBO) qd po for $26 \mathrm{wks}$. Endpoints included daily pt rating of abdominal pain at its worst during the previous 24 $\mathrm{h}$ on an 11-point scale ( $0=$ none, $10=$ very severe $)$ and CSBM frequency. Pts also rated adequate relief of IBS symptoms (yes/no) and IBS severity (5-point scale: $1=$ none, 5 = very severe) weekly. An Adequate Relief Responder was defined as a pt with adequate relief of IBS symptoms for $\geq 13$ of the 26 wks of the Treatment Period. Spearman correlations were performed for Adequate Relief Responders and individual symptom improvement.

Results The study included 401 LIN- and 403 PBO-treated pts. With LIN, $49 \%$ of pts were Adequate Relief Responders vs $25 \%$ of PBO pts (difference 24.1; $p<0.0001$; No. needed to treat 4.2). For 26 Wk IBS severity: $48 \%$ of LIN pts rated IBS severity as "none/mild" vs $26 \%$ of PBO pts (Table). In all pts, Adequate Relief Responders had a 26 -wk mean decrease in abdominal pain of $52 \%$ vs $18 \%$ for nonresponders and an increase from baseline in 26-wk mean CSBM frequency of $3.0 /$ wk vs $0.8 /$ wk for nonresponders. Improvements in abdominal pain $(r=0.48)$ and CSBM frequency $(r=0.53)$ correlated with adequate relief.

\section{Abstract PWE-026 Table}

\begin{tabular}{llll}
\hline & \multicolumn{2}{l}{ Pretreatment Period } & \multicolumn{2}{l}{ Treatment Period } \\
\cline { 3 - 4 } IBS severity, \% pts (Wk 26, LOCF) & & LIN ( $\mathbf{n = 4 0 1 )}$ & PBO ( $\mathbf{n}=\mathbf{4 0 3 )}$ \\
\hline None/Mild & 2.1 & 48.1 & 25.8 \\
Moderate & 24.3 & 31.7 & 38.7 \\
Severe/very severe & 73.6 & 20.2 & 35.5 \\
\hline
\end{tabular}

ITT population; LOCF, last observation carried forward

Conclusion Significantly more LIN pts vs $\mathrm{PBO}$ pts reported adequate relief of IBS symptoms and improvement of IBS severity during 26 wks of treatment. Gains in the \% of pts reporting adequate relief correlated strongly with improvements in abdominal pain and CSBM frequency.

Supported by Ironwood Pharmaceuticals Inc and by Forest Laboratories Inc. Editing assistance was provided by Complete Medical Communications, funded by Almirall.

Disclosure of Interest E. Quigley Speaker bureau with: Danone, Janssen, Procter and Gamble, sanofi-aventis, Shire and Yakult, Conflict with: Advisory boards for Almirall, Ironwood, Janssen, Norgine, Salix and Shire/Movetis, W. Chey Consultant for: Ironwood Pharmaceuticals and Forest Research Institute, B. Lavins Shareholder of: Ironwood Pharmaceuticals, Employee of: Ironwood Pharmaceuticals, M. Baird Shareholder of: Ironwood Pharmaceuticals, Employee of: Ironwood Pharmaceuticals, D. Fitch Shareholder of: Ironwood Pharmaceuticals, Employee of: Ironwood Pharmaceuticals, X. Hao Shareholder of: Ironwood Pharmaceuticals, Employee of: Ironwood Pharmaceuticals, K. Shi Employee of: Forest 